\title{
Lateral epicondylitis: A novel non-invasive treatment approach
}

\section{Christopher Bui}

Department of Physical Medicine and Rehabilitation, West Los Angeles Veterans Administration/UCLA, Los Angeles, CA, USA

\section{Email address:}

chrisbui.ucla@gmail.com

\section{To cite this article:}

Christopher Bui. Lateral Epicondylitis: A Novel Non-Invasive Treatment Approach. American Journal of Sports Science. Vol. 2, No. 5, 2014, pp. 111-114. doi: 10.11648/j.ajss.20140205.11

\begin{abstract}
Objective: To evaluate the effectiveness of the TheraPoint Focal Pressure Support (TP-FPS) as a non-invasive treatment for pain associated with lateral Epicondylitis (LE). Design: Prospective cohort study. Participants: $n=25$ study subjects (20 males and 5 females) ranging in age from 18-65 with pain in one upper limb either dominant (23) or non-dominant (2) associated with lateral epicondylitis for a minimum of six months. Interventions: Study participants were tasked to wear the TP-FPS for 3 hours per day for two weeks except when bathing or during main sleep hours. Outcome measures: Visual analog scale (VAS) pain score pre- and post-treatment period. Results: The combined (male and female) cohort average pre-treatment VAS pain score was $7.44+/-0.57$. Post-treatment VAS pain score was $1.07+/-0.42$, with a total reduction of 6.37 points and reached significance with $\mathrm{p}=0.0005$. Male cohort demonstrated an average pre-treatment VAS pain score of $7.45+/-0.56$. Post-treatment VAS pain score was $1.40+/-0.45$, with a total reduction of 6.05 points and reached significance with $\mathrm{p}=0.006$. Female cohort displayed an average pre-treatment VAS pain score of $7.40+/-1.92$. Post-treatment VAS pain score was $1.70+/-1.14$, with a total reduction of 5.70 and reached significance with $p=0.001$. No statistically significant difference was observed in the VAS pain score reduction between the male and female groups. Conclusion: The TP-FPS may indeed be a non-invasive therapeutic option for reducing pain associated with lateral epicondylitis.
\end{abstract}

Keywords: Lateral Epicondylitis, Elbow Pain, Non-Invasive, Pain Management, Pain Reduction

\section{Introduction}

Lateral epicondylitis (LE), otherwise known as tennis elbow, is a common clinical complaint with an incidence of $4-7 / 1000$ patients per year $[4,8,11]$. It is characterized by pain over the lateral humeral epicondyle, and commonly radiates into the dorsal forearm. This condition tends to be self-limiting, often resolving in 6-12 months irrespective of treatment; however, complaints may sometimes last up to two years or longer.

Although an exact cause on how lateral epicondylitis develops has yet to be determined, it is generally agreed that the overuse of wrist and hand functions contributes to the complaint of pain. Tissue-based pathology seen in cases of lateral epicondylitis includes degenerative changes at the proximal common extensor origin. In addition, myofascial trigger points in the muscles attached to the lateral epicondyle may also be a source of pain [6].

As described by Nirschl et al., workplace activities contribute to $35-64 \%$ of all cases [12]. Sports related injury is also a frequent culprit. This places a significant economic burden resulting in a high rate of missed workdays and productivity.

Multiple approaches for the treatment of lateral epicondylitis have been described in the medical literature. Analgesic medications such as oral or topical non-steroidal anti-inflammatory to opioid drugs have been used to treat the pain associated with lateral epicondylitis. In addition, a number of physiotherapeutic approaches including manipulation, therapeutic ultrasound, iontophoresis, phonophoresis, and low-level laser treatment have been described. More invasive interventional methods like corticosteroid, prolotherapy, and regenerative biologic injections have also been described. However, such interventions have not been shown to have consistent long-term clinical benefit $[10,11]$.

The aim of this paper is to determine the efficacy of a simple mechanical intervention utilizing the TheraPoint Focal Pressure Support (TP-FPS) in the treatment of pain associated with lateral epicondylitis. 


\section{Physiology and Mechanism of Action}

The TheraPoint Focal Pressure Support (TP-FPS) provides focal pressure to the area of application. As mentioned prior, there are at least two major theories as to the cause of lateral epicondylitis. This brings to light the unique nature of the TP-FPS. The system is theorized to affect both pathophysiological etiologies of lateral epicondylitis:

1. Active focal pressure for myofascial trigger point [6] and

2. Proximal isometric stabilization of the common extensor tendon, specifically the extensor carpi radialis brevis (ECRB) tendon, to curtail further overuse injury $[2,3,7,11]$.

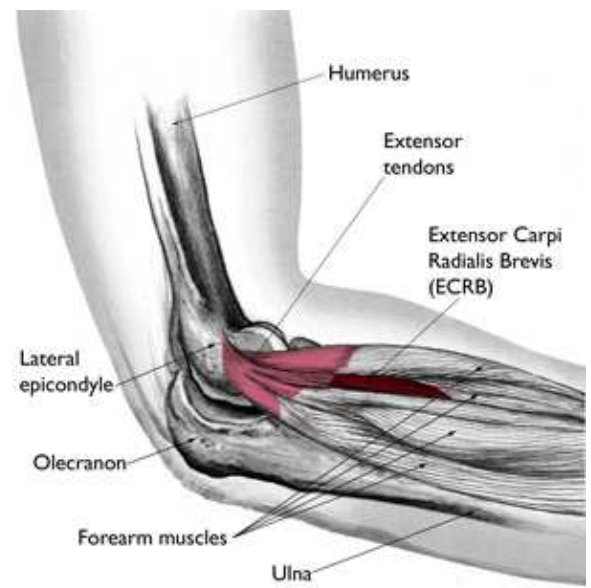

Figure 1. Origin of Extensor Tendons. The Body Almanac. (C) American Academy of Orthopedic Surgeons, 2003

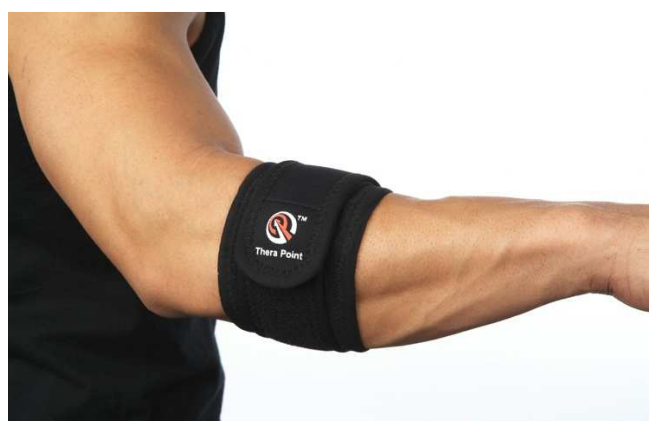

Figure 2. Application of TP-FPS

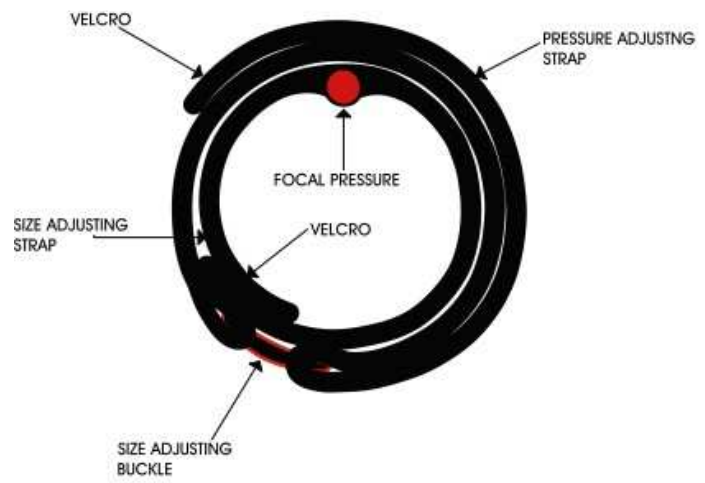

Figure 3. TP-FPS Cutaway Schematic

\section{Methods}

This was a prospective cohort study that took place over the course of six months.

Participants were recruited throughout the community. The following inclusion criteria was used: males and females between the ages of 18 and 65 , pain in one dominant or non-dominant upper limb associated with lateral epicondylitis for a minimum of six months, and have no significant impairment in the use of the affected upper limb.

Exclusion criteria included: potential participants actively receiving physiotherapy for their lateral epicondyle pain, recent $(\leq 6 \mathrm{mo})$ interventional injection, history of surgery to the elbow region, significant functional impairment in the affected upper limb, and/or significant analgesic medication use.

Once participants were deemed eligible they were given a TP-FPS to be used for a two-week treatment period. Participants were trained on donning and doffing the support. They were to wear the support for three hours per day, and not during bathing and main sleep hours.

35 participants were screened and accepted into the study. 30 participants were included in the final analysis with 5 being lost to follow-up.

Table 1. Subject Demographics

\begin{tabular}{lll}
\hline & Male & Female \\
\hline $\mathrm{N}$ & 20 & 5 \\
Median Age & 44.5 & 48 \\
Avg Duration of Pain Complaint (months) & $11.4+/-6$ & $9.49+/-5.1$ \\
Work:Sports Etiology & 0.67 & 1.5 \\
\hline
\end{tabular}

\subsection{Measurements}

Primary Outcome: Pre- and post-treatment visual analog scale (VAS) pain measurements were obtained and analyzed.

\subsection{Statistical Analysis}

SPSS Statistics ver. 20.0.1 was used for data analysis. Data was reported as means and standard deviations, medians and ranges. Two-sided, two-sample t-tests were used to compare pre- and post-treatment pain VAS scores with a significance level of 0.05 . Cohort was analyzed as a whole. ANOVA was utilized in the Male-to-female group comparison.

\section{Results}

30 participants completed the two-week treatment period with the TP-FPS. In the combined (male and female) cohort the average pre-treatment VAS pain score was $7.44+/-0.57$. Post-treatment VAS pain score was $1.07+/-0.42$, with a total reduction of 6.37 points and reached significance with $\mathrm{p}=0.0005$.

Male cohort demonstrated an average pre-treatment VAS pain score of $7.45+/-0.56$. Post-treatment VAS pain score was $1.40+/-0.45$, with a total reduction of 6.05 points and reached significance with $\mathrm{p}=0.006$. 
Female cohort displayed an average pre-treatment VAS pain score of $7.40+/-1.92$. Post-treatment VAS pain score was $1.70+/-1.14$, with a total reduction of 5.70 and reached significance with $\mathrm{p}=0.001$.

Post-hoc analysis between the male and female group did not show significance between the magnitudes of reduction in their VAS pain scores.

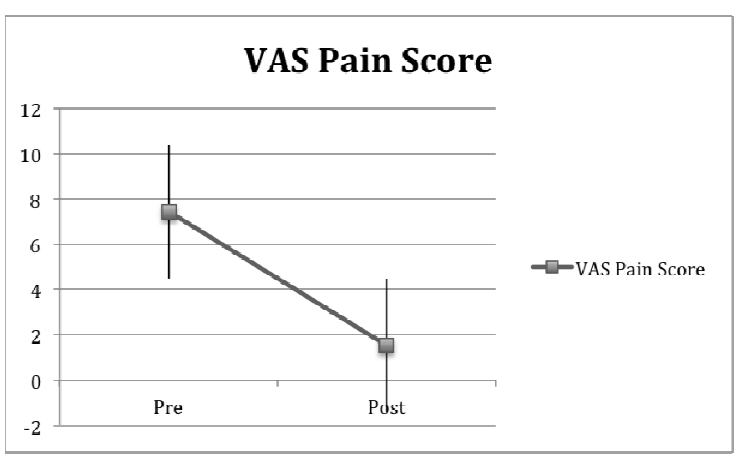

Graph 1. Changes in VAS Pain Scores Pre-Post-Treatment

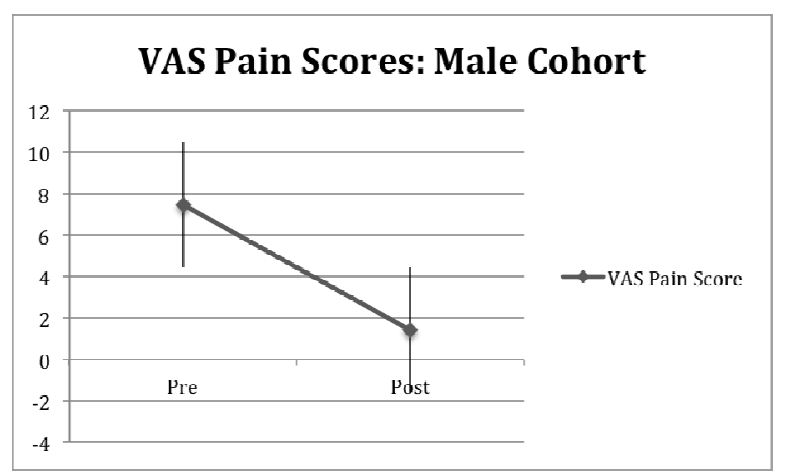

Graph 2. Changes in VAS Pain Scores Pre-Post-Treatment in the Male Cohort

VAS Pain Score: Female Cohort

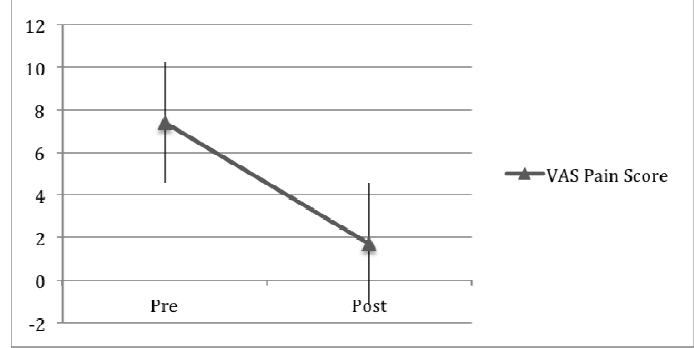

Graph 3. Changes in VAS Pain Scores Pre-Post- Treatment in the Female Cohort

\section{Discussion}

Lateral epicondylitis is a common clinical complaint, affecting $1-3 \%$ of the general population each year. A variety of treatments ranging from non-invasive physiotherapy techniques to analgesic medication and invasive therapies have been described in the medical literature. Most of these treatments exhibit significant pain reduction in the short term; however, lasting benefits are less clear.
The TheraPoint Focal Pressure Support aims to be a non-invasive treatment approach to reduce pain associated with lateral epicondylitis. The theorized mechanism of action includes both proximal stabilization of the wrist extensor tendons attaching at the lateral epicondyle, more specifically the ECRB tendon, as well as active focal pressure over the myofascial trigger point. It has been well studied that steadying the ECRB tendon lends to improvement in pain by limiting the amount of shear over the lateral epicondyle [18].

With regards to acupressure, there are a limited number of studies conducted on the effects of it on musculoskeletal pain, with most focusing on low back pain. According to Hsieh, et al. acupressure treatment six times over a one month period was significantly more effective in reducing chronic low back pain compared to a comparison group that was treated with physical therapy $(n=146)$ [19]. In a subsequent study, the Hsieh group found that acupressure significantly reduced chronic low back pain when compared to a placebo acupressure group $(n=129)$ [20]. Although the exact mechanism of acupressure has not been established, the dominant rationale is that it stimulates the release of neurochemicals associated with analgesia (i.e. endogenous opioid including ß-endorphins, enkephalins, dynorphins, and/or serotonin) [21]. Given that the elbow is also a component of the musculoskeletal system like the lumbar spine, it would be reasonable to extrapolate the analgesic effects of acupressure on the elbow.

This study demonstrated that use of the TheraPoint Focal Pressure Support was effective in reducing the VAS pain score of study participants. Post-treatment VAS pain scores decreased $86 \%, \mathrm{p}=0.0005$. No significant difference is pain score was observed between the male and female groups. Therefore, the TP-FPS may indeed be a viable non-invasive therapeutic option for reducing pain associated with lateral epicondylitis.

Limitations of this study include a small sample size and limited power. In addition, the absence of follow-up after the treatment period makes it difficult to determine long-term pain improvement. Participants were to only use the TP-FPS for the treatment of their pain. As a result, it is unknown if the TP-FPS has a synergistic effect with other non-invasive and invasive treatments. Lastly, our primary outcome was reduction in VAS pain score, which may provide us with a limited profile of the pain experienced by our study participants. Further studies are needed to evaluate and characterize the effectiveness of non-invasive therapeutic treatments, specifically the TP-FPS with regards to pain reduction in lateral epicondylitis.

\section{Conclusion}

Lateral epicondylitis, also known as tennis elbow, is a commonly seen musculoskeletal complaint. There are multiple approaches in the treatment of lateral epicondylitis. These include but are not limited to analgesic medication, physiotherapeutic modalities, and invasive interventions. 
This study demonstrated that the TheraPoint Focal Pressure Support may indeed be an effective non-invasive therapeutic option for reducing pain associated with lateral epicondylitis. Further studies are needed to evaluate and characterize the utility of non-invasive therapeutic treatments, specifically the TP-FPS in the treatment of lateral epicondylitis.

\section{References}

[1] Ajimsha, M. S., Chithra, S., \& Thulasyammal, R. P. (2012). Effectiveness of myofascial release in the management of lateral epicondylitis in computer professionals. Arch Phys Med Rehabil, 93(4), 604-609. doi: 10.1016/j.apmr.2011.10.012

[2] Ashe, M. C., McCauley, T., \& Khan, K. M. (2004). Tendinopathies in the upper extremity: a paradigm shift. $J$ Hand Ther, 17(3), 329-334. doi: 10.1197/j.jht.2004.04.002

[3] Blanchette, M. A., \& Normand, M. C. (2011). Augmented soft tissue mobilization vs natural history in the treatment of lateral epicondylitis: a pilot study. $J$ Manipulative Physiol Ther, 34(2), 123-130. doi: 10.1016/j.jmpt.2010.12.001

[4] Chard, M. D., \& Hazleman, B. L. (1989). Tennis elbow--a reappraisal. Br J Rheumatol, 28(3), 186-190.

[5] Chaudhury, S., de La Lama, M., Adler, R. S., Gulotta, L. V., Skonieczki, B., Chang, A., . . . Fealy, S. (2013). Platelet-rich plasma for the treatment of lateral epicondylitis: sonographic assessment of tendon morphology and vascularity (pilot study). Skeletal Radiol, 42(1), 91-97. doi: 10.1007/s00256-012-1518-y

[6] Chop, W. M., Jr. (1989). Tennis elbow. Postgrad Med, 86(5), 301-304, 307-308.

[7] Gabel, G. T. (1999). Acute and chronic tendinopathies at the elbow. Curr Opin Rheumatol, 11(2), 138-143.

[8] Hamilton, P. G. (1986). The prevalence of humeral epicondylitis: a survey in general practice. $J R$ Coll Gen Pract, 36(291), 464-465.

[9] Jensen, M. P., Turner, J. A., Romano, J. M., \& Fisher, L. D. (1999). Comparative reliability and validity of chronic pain intensity measures. Pain, 83(2), 157-162.
[10] Khan, K. M., Cook, J. L., Bonar, F., Harcourt, P., \& Astrom, M. (1999). Histopathology of common tendinopathies. Update and implications for clinical management. Sports Med, 27(6), 393-408.

[11] Kivi, P. (1983). The etiology and conservative treatment of humeral epicondylitis. Scand J Rehabil Med, 15(1), 37-41.

[12] Nirschl, R. P. (1992). Elbow tendinosis/tennis elbow. Clin Sports Med, 11(4), 851-870.

[13] Nourbakhsh, M. R., \& Fearon, F. J. (2008). The effect of oscillating-energy manual therapy on lateral epicondylitis: a randomized, placebo-control, double-blinded study. J Hand Ther, 21(1), 4-13; quiz 14. doi: 10.1197/j.jht.2007.09.005

[14] Overend, T. J., Wuori-Fearn, J. L., Kramer, J. F., \& MacDermid, J. C. (1999). Reliability of a patient-rated forearm evaluation questionnaire for patients with lateral epicondylitis. J Hand Ther, 12(1), 31-37.

[15] Rompe, J. D., Overend, T. J., \& MacDermid, J. C. (2007). Validation of the Patient-rated Tennis Elbow Evaluation Questionnaire. J Hand Ther, 20(1), 3-10; quiz 11. doi: 10.1197/j.jht.2006.10.003

[16] Shiri, R., Viikari-Juntura, E., Varonen, H., \& Heliovaara, M. (2006). Prevalence and determinants of lateral and medial epicondylitis: a population study. Am J Epidemiol, 164(11), 1065-1074. doi: 10.1093/aje/kwj325

[17] Simons, D. G., Hong, C. Z., \& Simons, L. S. (2002). Endplate potentials are common to midfiber myofacial trigger points. Am J Phys Med Rehabil, 81(3), 212-222.

[18] Walz, D. M., Newman, J. S., Konin, G. P., \& Ross, G. (2010). Epicondylitis: pathogenesis, imaging, and treatment. Radiographics, 30(1), 167-184. doi: 10.1148/rg.301095078

[19] Hsieh, L.L., et al., Treatment of low back pain by acupressure and physical therapy: randomised controlled trial. $B M J, 2006.332(7543)$ : p. 696-700.

[20] Hsieh, L.L., et al., A randomized controlled clinical trial for low back pain treated by acupressure and physical therapy. Prev Med, 2004. 39(1): p. 168-76.

[21] Luo, D., X. Wang, and J. He, A comparison between acute pressure block of the sciatic nerve and acupressure: methodology, analgesia, and mechanism involved. $J$ Pain Res, 2013. 6: p. 589-93. 